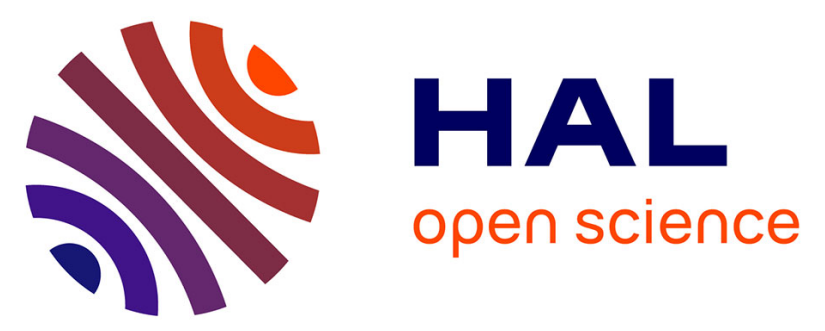

\title{
Optimization of ion trajectories in a dynamically harmonized Fourier-Transform Ion Cyclotron Resonance cell using a Design of Experiments strategy
}

Julien Maillard, Justine Ferey, Christopher P. Rüger, Isabelle Schmitz-Afonso, Soumeya Bekri, Thomas Gautier, Nathalie Carrasco, Carlos Afonso, Abdellah Tebani

\section{To cite this version:}

Julien Maillard, Justine Ferey, Christopher P. Rüger, Isabelle Schmitz-Afonso, Soumeya Bekri, et al.. Optimization of ion trajectories in a dynamically harmonized Fourier-Transform Ion Cyclotron Resonance cell using a Design of Experiments strategy. Rapid Communications in Mass Spectrometry, 2020, 34 (7), pp.e8659. 10.1002/rcm.8659 . insu-02395300

\section{HAL Id: insu-02395300 \\ https://hal-insu.archives-ouvertes.fr/insu-02395300}

Submitted on 8 Mar 2021

HAL is a multi-disciplinary open access archive for the deposit and dissemination of scientific research documents, whether they are published or not. The documents may come from teaching and research institutions in France or abroad, or from public or private research centers.
L'archive ouverte pluridisciplinaire HAL, est destinée au dépôt et à la diffusion de documents scientifiques de niveau recherche, publiés ou non, émanant des établissements d'enseignement et de recherche français ou étrangers, des laboratoires publics ou privés. 
1 Optimization of ion trajectories in a dynamically harmonized Fourier-Transform Ion Cyclotron

6 Julien MAILLARD ${ }^{1,2}$, Justine FEREY ${ }^{2}$, Christopher P. Rüger ${ }^{2}$, Isabelle SCHMITZ-

7 AFONSO $^{2}$, Soumeya BEKRI ${ }^{3}$, Thomas GAUTIER ${ }^{1}$, Nathalie CARRASCO ${ }^{1}$, Carlos

$8 \mathrm{AFONSO}^{2}$ and Abdellah TEBANI ${ }^{2.3 *}$

9

$10{ }^{1}$ LATMOS/IPSL, Université Versailles St Quentin, UPMC Université Paris 06, CNRS, 11

11 blvd d'Alembert, F-78280 Guyancourt, France

$12{ }^{2}$ Université de Rouen, Laboratoire COBRA UMR 6014 \& FR 3038, IRCOF, 1 Rue

13 Tesnière, 76821 Mont St Aignan Cedex, France

$14{ }^{3}$ Department of Metabolic Biochemistry, Rouen University Hospital, Rouen, 76000, 15 France 


\section{Abstract}

18 The optimization of the ion trajectories in the ion cyclotron resonance (ICR) cell of a

19 Fourier transform ICR mass spectrometer is a crucial step to obtain the best dynamic range,

20 mass resolution, and mass accuracy. With the recent introduction of the dynamically

21 harmonized cell, the complexity of tuning expanded drastically, and a fine-tuning of the

22 DC voltages is required to optimize the ion cloud movement. This adjustment is typically 23 performed manually.

24 Here, we propose a computational method based on a design of experiments (DoE) strategy 25 to overcome the limits of classical manual tuning. This DoE strategy was exemplarily 26 applied on a 12T FTICR equipped with a dynamically harmonized ICR cell. The chemometric approach, based on a composite central face design (CCF), was first applied on a reference material (sodium trifluoroacetate) allowing for the evaluation of the primary cell parameters. Eight factors were identified related to shimming and gating. The summed 30 intensity of the signal corresponding to the even harmonics was defined as one quality 31 criteria.

32 Consequently, the DoE response allowed for rapid and complete mapping of cell parameters resulting in an optimized parameter set. The new set of cell parameters was

34 applied to the study of an ultra-complex sample. Here, Tholins, an ultra-complex mixture that mimics the haze present on Titan, was chosen. We observed a substantial improvement

36 in mass spectrometric performance. The sum of signals related to harmonics was decreased

37 by a factor of three (from $4 \%$ for conventional tuning to $1.3 \%$ ). Furthermore, the dynamic range was also increased and lead to an increase of attributed peaks by $13 \%$. 

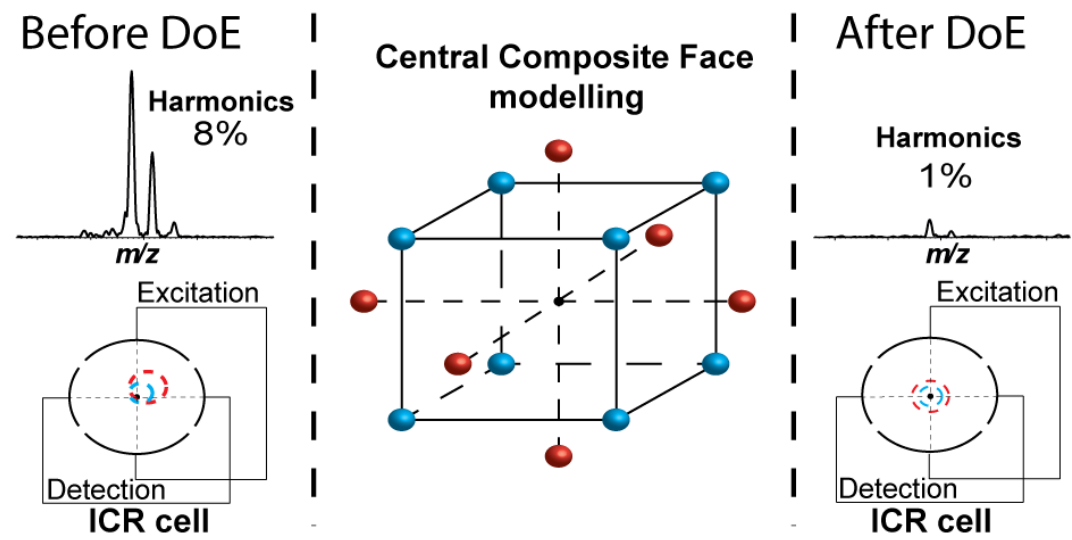


\section{1. Introduction}

43 Fourier Transform Ion Cyclotron Resonance mass spectrometer (FTICR) is well known to

44 be the most powerful mass spectrometer by reaching unprecedented resolving power, mass accuracy and sensitivity levels ${ }^{1,2}$. It turned out to be the most appropriate instrument for the characterization of ultra-complex mixtures such as petroleum ${ }^{3-7}$, natural organic matter ${ }^{8}$ or extraterrestrial organic matter ${ }^{9,10}$. In order to detect the cyclotron motion, FTICR uses the combination of a static electric field which is hyperbolic near the center and a strong homogeneous static magnetic field (up to 21 Tesla - so far). The combination of both fields retains ions in the cell. Ions in the magnetic field travel according to a cyclotron motion defined by equation 1 .

$$
\omega_{0}=\frac{q B_{0}}{m}
$$

Equation 1: Cyclotron frequency $\left(\omega_{0}\right)$ of a charged particle in a homogeneous magnetic field. $q$ is the charge of the particle, $B_{0}$ the strength of the magnetic field and $m$ the mass of the particle.

In addition, the ion packet undergoes a drift of a trajectory equal to $\mathbf{E x B}$ where $\mathrm{E}$ is the electric field and B is the magnetic field. Both of these movements allow the ion packet to be trapped in the cell ${ }^{2,11}$.

60 The shape of the ICR cell was improved steadily over time from first cubic geometry ${ }^{12}$ to 61 a variety of spherical designs varying in electrode shape and number ${ }^{13}$. As an example, the 62 orthorhombic capacitively coupled cell developed by Steven Beu in $1991^{14}$ or the infinity 63 cell which was designed by Caravatti et al. in 1991 are cited here ${ }^{15}$, the later remaining the 64 most commonly used nowadays. In recent instruments, the so-called dynamically 65 harmonized cell is frequently deployed ${ }^{2}$. For the purpose of radiofrequency excitation and 66 detection of ions, the cell is composed of several electrodes. In case of the dynamically 
67 harmonized cell, these excitation and detection electrodes are specially shaped to compensate the quadrupolar electric field over the entire cell's body. It allows to produces a volume where, over the orbit of the ions, the average field is quadrupolar at all cyclotron radii. Thus, the frequency drifts of ions of the same mass over time along the $\mathrm{Z}$ axis are reduced. This makes possible to record transients for a longer time than in the previous cells and thus significantly improve performance

Optimized detection in terms of highest resolving power, sensitivity and dynamic range is obtained when the ion cloud is perfectly centered in the middle of the magnet. However, in practice, the imperfections of the alignment cause a bias in the position of the ion packets and thus a deviation in the cyclotron motion synchronization. This issue is solved by adding another set of segments to the dynamically harmonized cell in order to smooth imperfections by applying additional DC (Direct Current) voltages ${ }^{11}$. With this approach, it is possible to decrease the abundance of even-harmonic signals and, thus, to minimize magnetron motion. However, the values for the additionally applied DC voltages have to be tuned manually, which requires specially trained personnel and is tedious and timeconsuming. In the present work, an experimental design approach is proposed in order to overcome the drawbacks of the manual ICR cell tuning. The approach seeks to optimize the DC voltages and reach the best set of parameters. Design of experiments (DoE) is a rigorous procedure in which defined modifications are induced based on a given set of variables. The response of the system is recovered to create a mathematical model, which allows a prediction of a set of parameters optimizing the response value ${ }^{16-18}$. Some example of mass spectrometer's optimization using DoE can be cite, such as the work performed by Lemonakis et al (2016) and Tebani et al (2016) ${ }^{19,20}$. Here, this method is applied on a defined response, the sum of the abundance of the second harmonic of sodium trifluoroacetate cluster. 
92 After optimization, the obtained parameters are applied to the analysis of a complex

93 mixture, Tholins. This synthetic material is used to understand the chemistry occurring in

94 Titan's atmosphere by artificially mimicking the brown haze surrounding the largest moon

95 of Saturn ${ }^{21-25}$. More importantly, Tholins have shown to be ultra-complex mixtures with

96 high isobaric complexity and thousands of elemental compositions ${ }^{26}$. Tholins spectra

97 obtained after DoE and after the usual manual method are compared. The potential of the

98 developed method for complex mixtures analysis is highlighted evaluating the mass

99 spectrometric performance, i.e., resolving power, the abundance of harmonics and number

100 of detected signals.

101 


\section{Experimental methods}

103

104

105

106

107

108

109

110 in the plasma discharge was maintained at $0.9 \pm 0.1 \mathrm{mbar}$, and the reaction took place at

111 room temperature $(293 \mathrm{~K})$.

\subsection{Tholins production}

Tholins were produced following the PAMPRE procedure (French acronym for Aerosols

Microgravity Production by Reactive Plasmas) presented elsewhere ${ }^{24,27}$. Briefly, in a stainless steel tubular reactor, a continuous gas mixture composed of $95 \mathrm{v}-\%$ nitrogen and $5 \mathrm{v}-\%$ methane was injected through polarized electrodes and evacuated by a primary vacuum pump system. A Radio Frequency - Capacity Coupled Plasma (RF-CCP) discharge was established in the gas mixture deploying an RF of $13.56 \mathrm{MHz}$. The pressure

\subsection{Instrumentation}

\subsubsection{Fourier transform ion cyclotron resonance mass spectrometer}

114 All analyses were performed on a FTICR Solarix XR from Bruker equipped with a 12T 115 superconducting magnet and a dynamically harmonized ICR cell. The following ion 116 transfer parameters were used for both electrospray (ESI) and laser desorption ionization 117 (LDI) analyses in positive ion mode: capillary exit $150 \mathrm{~V}$, deflector plate $200 \mathrm{~V}$, funnel1 $118150 \mathrm{~V}$, skimmer1 25V, funnel RF amplitude 60Vpp, octopole frequency 5MHz, octopole 119 RF amplitude $350 \mathrm{Vpp}$, lower cut-off of the quadrupole at $\mathrm{m} / \mathrm{z} 120$, time-of-flight $0.7 \mathrm{~ms}$, 120 frequency TOF $6 \mathrm{MHz}$, TOF RF amplitude $200 \mathrm{Vpp}$, side kick offset $-1 \mathrm{~V}$, front and back 121 trapping plate $1.75 \mathrm{~V}$.

122 For ESI the following parameters were used: capillary $4.5 \mathrm{kV}$, spray shield $-500 \mathrm{~V}$, dry gas 123 flow $2 \mathrm{~L} \cdot \mathrm{min}^{-1}$, dry gas temperature $180^{\circ} \mathrm{C}$, nebulizer gas flow 0.5 bar. All analyses were 124 recorded on 20 scans with a quadrupole accumulation time of $0.1 \mathrm{~s}$. Electrospray spectra 
125 were recorded with a mass range from $\mathrm{m} / \mathrm{z}, 98$ to 1,200 and a transient length of $2.2 \mathrm{~s}$. Sodium

126 trifluoroacetate (NaTFA, Sigma Aldrich) at $0.1 \mathrm{mg} \cdot \mathrm{ml}^{-1}$ in $\mathrm{ACN} / \mathrm{H}_{2} \mathrm{O}(50 / 50, \mathrm{v} / \mathrm{v})$ was used

127 as a standard for the design of experiments approach.

128 Complex Tholins mixture analyses was recorded applying LDI. The solid sample was 129 deposed on a MALDI plate following previous work ${ }^{28}$. The third harmonic of a Nd:YAG 130 laser at $355 \mathrm{~nm}$ delivering a maximum output of $0.5 \mathrm{~mJ}$ (Smartbeam II, Bruker) was used to 131 ionize the samples with the following parameters: laser power $15 \%$, laser shots per scan 13250 , shots frequency $1 \mathrm{kHz}$, plate offset $100 \mathrm{~V}$. The recorded mass spectra combined 800 133 scans to achieve high signal-to-noise. LDI spectra were recorded with a mass range from $134 \mathrm{~m} / \mathrm{z} 110$ to 1200 and a transient length of $4.3 \mathrm{~s}$.

\subsection{Experimental design}

136 2.3.1. Software

137 DataAnalysis 4.4 (Bruker Daltonics $\mathrm{GmbH}$, Bremen) was used to process all mass 138 spectrometric analyses, including $\mathrm{m} / \mathrm{z}$-calibration and response recovering (abundance of 139 the harmonic signals).

140 MODDE 11 software (Umetrics, Sartorius Stedim Data Analytics AB, Umea, Sweden) was 141 used to perform the consecutive data analyses and modeling. The initial step is the 142 definition of the response (i.e., user observation). Then, in a second step, factors influencing

143 the response are defined. Afterward, several choices of experimental design shapes can be 144 chosen. The software will automatically create the DoE given to the user as a coded matrix 145 (Table 1) and a real matrix (Table S1). This matrix defines the experiments to be recorded 146 and from which the response has to be extracted. Then, MODDE propose an automatic

147 fitting method (e.g., partial least squares regression - PLS) allowing the visualization of the 
148 quality and the predictability of the DoE. Finally, the optimizer tool was used to predict the

149 set of parameters giving the best response result.

150

151

152

153

154

155

156

157

158

159

160

161

162

163

164

165

166

167

168

169

170

171

\subsubsection{Factorial designs}

The tuning of the ICR cell is established with eight principal parameters, four shimming parameters (shimming $0^{\circ}$, shimming $180^{\circ}$, shimming $90^{\circ}$ and shimming $270^{\circ}$ ) and four gating settings (gated $0^{\circ}, 90^{\circ}, 180^{\circ}$, and $\left.270^{\circ}\right)^{11}$. The set of gating parameters represents the values of DC voltages applied during the injection in the ICR cell in order to correctly trap the ion packets. The set of shimming parameters represents the values of DC voltages applied for the excitation and the detection of the ions packets in the ICR cell. A simplified description of the ICR cell is given in figure 1. Other parameters of the ICR cell are not included (e.g., back and front plate voltages, sidekick offset) as they are easily and rapidly manually tunable. This significantly reduces the number of experiments required for the DoE approach, considerably decreasing the optimization time.

Parameters were grouped in pairs leading to four couples of parameters: shimming $0^{\circ}-180^{\circ}$ (Shi0), shimming $90^{\circ}-270^{\circ}$ (Shi90), gated $0^{\circ}-180^{\circ}$ (Gat0) and gated $90^{\circ}-270^{\circ}$ (Gat90) simplifying the optimization process compared to the conventional manual procedure. The values of these couples change symmetrically, meaning that when the value of one part is increased, the other part decreases about the same value. Initially, all values are set to $1.5 \mathrm{~V}$ (default settings). As example, increasing the value of the shimming $0^{\circ}$ about $50 \mathrm{mV}$ to $1.55 \mathrm{~V}$ results at a value of the shimming $180^{\circ}$ of $1.45 \mathrm{~V}$. In addition to these four coupled parameters, the sweep excitation power (SEP) was added as a factor parameter for the first step of the optimization strategy. SEP has a crucial impact on the ion trajectories as it defines their radius. After defining the assessed experimental factors, it was necessary to define the desired response as a result of the optimization. Following the classical 
172 optimization method, the intensity ratio between the ion of the NaTFA at $m / z 702.86324$

173 and its second harmonic at $m / z 351.43164$ was recovered.

174 The optimization of the dynamically harmonized cell parameters was divided into two

175 steps. The first screening step was performed with wide parameter intervals to evaluate the

176 effects of each parameter on the defined response. The second part, named optimization

177 experimental design, focused on narrower intervals to reach the best set of parameters. The

178 applied chemometric approach for the two steps is based on a Composite Central

179 Composite face design (CCF) which takes into account interactions between parameters.

180 The coded CCF for the first step is given in Table $\mathbf{1}$ formatted according to the required

181 software input. Basically, the table reflects the conducted experimental plan, where -1

182 means that the parameter was set at the respective low level (e.g., for Shi 0 at 1.465V) and

$183+1$ at the respective high level. Defined intervals for each parameter are given in Table 2

184 for the screening and optimization step. These intervals have been defined quickly by

185 varying each of the parameters until the intensity of the harmonic reaches a minimum level.

186 This step takes about 5 minutes.

\section{2.3.3. Multivariate modeling}

188 Partial Least Squares regression (PLS) was used to model each CCF design and to find the

189 best set of parameters regarding the response chosen using the optimizer tool implemented

190 in MODDE 11. Thus, PLS coefficients are used to estimate factor effects. A positive value

191 of the coefficient indicates an increase of the response upon increasing the corresponding

192 parameter, whereas a negative value indicates a decrease of the response upon increasing

193 the corresponding parameter. The larger the absolute value of the PLS coefficient, the

194 higher the effect of the parameter on the response. Analysis of variance (ANOVA) applied

195 to the sum of squares of the PLS regression coefficient was used to assess the significance 
196 of the factor effect. For a statistically significant effect of a factor, the model $p$-value of

197 ANOVA should not exceed $0.05^{29}$.

198 3. Results and discussion

199

\subsection{Screening experimental design using NaTFA}

200

201

202

203

204

205

206

207

208

The design of experiments approach was performed using NaTFA to minimize the abundance of signals related to harmonics. Briefly, the influence of four pairs of factors and the SEP (Shi0, Shi90, Gat0, Gat90, and Sweep Excitation Power) was evaluated using an even harmonic peak intensity of the ion $\mathrm{m} / \mathrm{z} .702 .86324$ at $\mathrm{m} / \mathrm{z} 351.43164$ as the response. A Central Composite Face design (CCF) was chosen as it is generally applicable for this type of optimization problems.

Figure 2a visualizes the effect of the different factors on the even harmonic abundance in the screening step. SEP, Shi0, and Shi90 revealed the most significant effects on the intensity of the second harmonic peak, with contribution factors of $28.0 \%, 15.4 \%$, and $33.3 \%$ respectively. SEP played a positive effect with high values leading to high abundance of the harmonic. In contrast, Shi0 and Shi90 showed a negative response, with high values resulting in low abundance of the harmonic. In addition, the interaction of Shi0 and Shi90 provided a significant positive effect, which is related to a high separation effect of these parameters. Hence these two couples of parameters have two very different effects on the abundance of the harmonic and, thus, on the ion cloud trajectories.

The prediction plot obtained based on the 27 experimental data points, between the predicted and observed values of the abundance of the second harmonic, is shown in Fig. $\mathbf{2 b}$. This representation allows to access the quality of prediction with this model based on the initial data set. The cumulative modeled variation $\left(\mathrm{R}^{2} \mathrm{X}=99.1 \%\right)$ and the cumulative predicted variation $\left(\mathrm{Q}^{2} \mathrm{Y}=62.8 \%\right)$ reflect the explained variance and the predictability of 
220 the model. The near to one $\mathrm{R}^{2} \mathrm{X}$ value proves the validity of the model. The $\mathrm{Q}^{2} \mathrm{Y}$ value

221 (which is above 50\%) proves that the model can give predictable values of parameters for

222 the defined response ${ }^{17,18,29}$. However, this value could be improved to obtain better

223 optimization results, which will be the aim of the following part of the optimization

224 experimental design. The values of the regression coefficients and their respective $p$-values

225 are given in Table S-3 (Supplementary data). The fitted models' metrics, ANOVA and

226 validation results of each model are presented in Tables S-4 and S-5 (Supplementary data).

227 Using this built model, the instrumental parameters were optimized to reach the lowest

228 harmonic intensity. The predicted optimized values are presented in Table 2. Nevertheless,

229 the obtained set of predicted values reach the extremity of each defined interval. Therefore,

230 an additional optimization step was performed focusing on tight intervals for Shi0, Shi90,

231 Gat0, and Gat90. It should be noted that the SEP and shimming values are two decorrelated

232 parameters. In the rest of this study, the SEP was fixed at a value of $30 \%$ in order to focus

233 on fine tuning. This value is a percentage of the maximum excitation amplitude.

\subsection{Optimization experimental design using NaTFA}

As described above, for this step, the sweep excitation power (SEP) was removed from the studied factors to focus only on the cell parameters. It turned out, that Shi0 and Shi90 had a major negative response on the second harmonic abundance (Fig. 2c), which is consistent with the results obtained in the previous screening step. Coded regression coefficients, ANOVA and model validation results are given in Tables S-2, S-5, and S-6

240 (Supplementary data). The fitted model shows a cumulative modeled variation of $\mathrm{R}^{2} \mathrm{X}=$ $24199.5 \%$ and a cumulative predicted variation of $\mathrm{Q}^{2} \mathrm{Y}=88.9 \%$ as showed in Fig. $2 \mathrm{~d}$. These

242 results improved compared to the screening step and proved the high predictability of the 
244 optimized set of parameters provided an abundance of $1.3 \%$ of second harmonics compared

245 to the base signal. In comparison, the non-optimized parameters (manufacturer default

246 values) provided $8 \%$ and manually optimized parameters provided $4 \%$ of harmonic

247 abundance, respectively.

248 This first study proved the possible optimization of the movement of ions in the ICR cell

249 by lowering harmonic peaks. This DoE methodology was validated by the study of a

250 NaTFA standard solution typically used for instrument calibration and optimization.

251 However, the high interest of optimizing the ion trajectories in the ICR cell is related to the

252 description of complex mixtures with unknown constituents. Consequently, the set of 253 parameters yielded by the DoE methodology have been applied to study Titan tholins. The 254 results were compared to those obtained after the manual optimization.

\subsection{Application for the analysis of complex mixtures: Titan tholins}

256 The largest moon of Saturn, Titan, is surrounded by a thick and nitrogen-rich fog with some 257 fraction of methane $\mathrm{e}^{30-33}$. Understanding how this haze is produced along with its molecular 258 composition are crucial steps from a planetary and prebiotic chemistry perspective $\mathrm{e}^{34,35}$. 259 Tholins are analogs of Titan's haze. They are laboratory-produced and used as a proxy on 260 Earth as there is currently no sample return option. This material is an extremely complex 261 mixture comparable to the complexity of petroleum and as such ideal for evaluating the 262 tuning of the dynamically harmonized cell parameters ${ }^{26}$. Figure 3 shows an overview of a 263 tholins mass spectrum, containing more than 50,000 peaks. This mass spectrum was 264 generated with LDI ionization together with the optimized cell tuning parameters resulting 265 from the DoE approach on NaTFA. The insert in Figure 3 presents the molecular 266 complexity on a single nominal mass revealing more than 100 resolved peaks. In this study, 
267 we used this ultra-complex sample to compare the effects of the above-described optimization strategy with the conventional method relying on manual optimization.

269 The comparison between the detected species applying the parameters given by manual 270 tuning and DoE optimization is illustrated in Figure 4. The mass spectrum at the top was 271 recorded after the conventional manual optimization of the ICR cell. This manual optimization can take several hours easily to obtain high performance due to the manual iteration process. The spectrum at the bottom was recorded after deploying the optimized parameter set given by the newly developed DoE strategy, which takes approximately one hour. It should be mentioned that the shimming parameters are re-optimized once a year to compensate instrumental's drift. Except for the cell parameters, the two spectra were recorded with the exact same conditions. The number of detected species is significantly higher with the DoE optimization. Figure 4, giving a small fraction of the mass spectrometric complexity revealed thirteen additional species (indicated with a red star) for the DoE optimized spectrum compared to the spectrum resulted after manual tuning. The attribution of these new peaks is given in table S-7. This observation can be induced by two effects: 1) The ion packets is more centered in the cell thanks to the computational method, inducing a higher dynamic range or, 2) in the spectrum after manual adjustment, some peaks are in coalescence with their close neighbor. This effect is not present after the DoE tuning, which explain why more peaks are observed in this case. In order to reinforce this, through attributions, it can be justified that these peaks are not harmonics. Manual optimization is a difficult and limited process. Indeed, it is impossible for the human eye to take into account the interactions between the various parameters. Therefore, the location of the ions is not perfectly centered and could still be improved. Furthermore, this method

290 is a time-consuming iterative process based on trial and error steps to reach acceptable 291 values specified by the manufacturer (here below $6 \%$ for the second harmonic). The above 
described DoE strategy, performed with NaTFA, allows a complete mapping of the different parameter effects along with their interactions, which allows modeling of the system. Thus, this strategy leads to a more fine-tuning and a greater reduction of harmonics compared to the conventional manual attempt.

Different metrics obtained for DoE originated and manually recorded spectra are listed in Table 3. These results were obtained after recording 5 replicates for each tuning. As illustrated in Figure 4 applying the DoE generated parameters significantly increased the number of observed peaks. The comparison with the manually tuned cell revealed $13 \%$ more peaks attributed after the DoE tuning. The resolving power is also slightly increased after the experimental design optimization: approx. 1,440,000 with the DoE method and 1,350000 with the manual method at $\mathrm{m} / \mathrm{z}$ 400. A slight increase is also observed for the average mass accuracy of the elemental composition assignment. All these results are summarized in Figure 5.

The application of the new predicted dynamically harmonized cell parameters on Titan's tholins showed a clear enhancement on almost every metric of the recorded spectrum in comparison with the spectrum generated with the non-tuned cell but also with the manually tuned cell. Furthermore, this work will be useful for the implementation of quadrupolar ion detection, which commonly induces higher order harmonics with increased abundance. This newly developed optimization strategy is highly time efficient compared to the conventional method, taking only one hour including the recording of the spectra, data processing, and data analysis.It is a universal approach and can be applied to any complex mixture such as petroleum and biological sample materials. Those ultra-complex mixtures usually raises high analytical challenges regarding experimental optimization. 


\section{4. Conclusions}

317 The developed design of experiments (DoE) strategy has proven to be applicable for

318 generating optimized parameter sets for a dynamically harmonized ICR cell. We could

319 show that the approach results in settings leading to a reduction of harmonics to levels not

320 reached by manual tuning and far below the manufacturer default values. Moreover, the

321 optimization process takes significantly less time and can be performed by any instrument

322 user. This substantial improvement allows for a better performance of the FTICR by

323 significantly increasing all metrics of the recorded spectrum, i.e., mass accuracy, resolution

324 and dynamic range, for both the standard as well as the ultra-complex mixture. Finally, this

325 computational procedure based on a composite face design could be applied for any other

326 mass spectrometric parameter optimization problem. The authors believe this will allow for

327 a more transparent and more structured design when performing method development. 


\section{5. Acknowledgments}

331 N.C. thanks the European Research Council for funding via the ERC PrimChem project 332 (grant agreement No. 636829.).

333 Financial support from the National FT-ICR network (FR 3624 CNRS) for conducting the 334 research is also gratefully acknowledged.

335 This work was also supported at COBRA laboratory by the European Regional 336 Development Fund (ERDF N³1708), the Région Normandie, and the Labex SynOrg 337 (ANR-11-LABX-0029) together with Normandie Université (NU), the Centre National de 338 la Recherche Scientifique (CNRS), Université de Rouen Normandie (URN) and Innovation 339 Chimie Carnot (I2C). 
1. Smith RD, Cheng X, Bruce JE, Hofstadler SA, Anderson GA. TRAPPING,

9. Schmitt-Kopplin P, Gabelica Z, Gougeon RD, et al. High molecular diversity of extraterrestrial organic matter in Murchison meteorite revealed 40 years after its fall. Proceedings of the National Academy of Sciences of the United States of America. 2010;107(7):2763-2768.

10. Schmitt-Kopplin P, Harir M, Kanawati B, Tziozis D, Hertkorn N, Gabelica Z. Chemical footprint of the solvent soluble extraterrestrial organic matter occluded in soltmany ordinary chondrite. Meteorites. 2012:79-92.

11. Jertz R, Friedrich J, Kriete C, Nikolaev EN, Baykut G. Tracking the Magnetron Motion in FT-ICR Mass Spectrometry. Journal of the American Society for Mass Spectrometry. 2015;26(8):1349-1366.

12. Guan S, Marshall AG. Ion traps for Fourier transform ion cyclotron resonance mass spectrometry: principles and design of geometric and electric configurations. International Journal of Mass Spectrometry and Ion Processes. 1995;146-147:261296.

13. Marshall AG, Hendrickson CL, Jackson GS. Fourier transform ion cyclotron resonance mass spectrometry: A primer. Mass Spectrometry Reviews. 1998;17(1):1-35.

14. Beu SC, Laude DA. Open trapped ion cell geometries for Fourier transform ion cyclotron resonance mass spectrometry. International Journal of Mass Spectrometry and Ion Processes. 1992;112(2-3):215-230. 
15. Caravatti $\mathrm{P}$, Allemann M. The 'infinity cell': A new trapped-ion cell with radiofrequency covered trapping electrodes for fourier transform ion cyclotron resonance mass spectrometry. Organic Mass Spectrometry. 1991;26(5):514-518.

16. Gerretzen J, Szymanska E, Jansen JJ, et al. Simple and Effective Way for Data Preprocessing Selection Based on Design of Experiments. Analytical chemistry. 2015;87(24):12096-12103.

17. Eliasson M, Rannar S, Madsen R, et al. Strategy for optimizing LC-MS data processing in metabolomics: a design of experiments approach. Analytical chemistry. 2012;84(15):6869-6876.

18. Eriksson L. Design of Experiments: Principles and Applications

Umetrics. 2008.

19. Lemonakis N, Skaltsounis AL, Tsarbopoulos A, Gikas E. Optimization of parameters affecting signal intensity in an LTQ-orbitrap in negative ion mode: A design of experiments approach. Talanta. 2016;147:402-409.

20. Tebani A, Schmitz-Afonso I, Rutledge DN, Gonzalez BJ, Bekri S, Afonso C. Optimization of a liquid chromatography ion mobility-mass spectrometry method for untargeted metabolomics using experimental design and multivariate data analysis. Analytica chimica acta. 2016;913:55-62.

21. Khare BN, Sagnan C, Zumberge JE, Sklarew DS, Nagy B. Organic Solids Produced by Electrical Discharge in Reducing Atmospheres Tholin Molecular Analysis. Icarus. 1981;48:290-297.

22. Coll P, Coscia D, Smith N, et al. Experimantal laboratory simulation of Titan's atmosphere: aerosols and gas phase. Planetary and Space Science. 1999;47:13311340 .

23. Somogyi A, Oh CH, Smith MA, Lunine JI. Organic environments on Saturn's moon, Titan: simulating chemical reactions and analyzing products by FT-ICR and ion-trap mass spectrometry. Journal of the American Society for Mass Spectrometry. 2005;16(6):850-859.

24. Szopa C, Cernogora G, Boufendi L, Correia JJ, Coll P. PAMPRE: A dusty plasma experiment for Titan's tholins production and study. Planetary and Space Science. 2006;54(4):394-404.

25. Imanaka H, Smith MA. Formation of nitrogenated organic aerosols in the Titan upper atmosphere. Proceedings of the National Academy of Sciences. 2010;107.

26. Maillard J, Carrasco N, Schmitz-Afonso I, Gautier T, Afonso C. Comparison of soluble and insoluble organic matter in analogues of Titan's aerosols. Earth and Planetary Science Letters. 2018;495:185-191.

27. Gautier T, Carrasco N, Buch A, Szopa C, Sciamma-O’Brien E, Cernogora G. Nitrile gas chemistry in Titan's atmosphere. Icarus. 2011;213(2):625-635.

28. Barrere C, Hubert-Roux M, Lange CM, et al. Solvent-based and solvent-free characterization of low solubility and low molecular weight polyamides by mass spectrometry: a complementary approach. Rapid communications in mass spectrometry : RCM. 2012;26(11):1347-1354.

29. Montgomery D. Design and Analysis of Experiments. John Wiley \& Sons. 2008.

30. Niemann HB, Atreya SK, Bauer SJ, et al. The abundances of constituents of Titan's atmosphere from the GCMS instrument on the Huygens probe. Nature. 2005;438(7069):779-784.

31. Fulchignoni M, Ferri F, Angrilli F, et al. In situ measurements of the physical characteristics of Titan's environment. Nature. 2005;438(7069):785-791. 
443

444
32. Lebreton JP, Witasse O, Sollazzo C, et al. An overview of the descent and landing of the Huygens probe on Titan. Nature. 2005;438(7069):758-764.

33. Israel G, Szopa C, Raulin F, et al. Complex organic matter in Titan's atmospheric aerosols from in situ pyrolysis and analysis. Nature. 2005;438(7069):796-799.

34. Sagan C. The origin of life in a cosmic context. Origins of Life. 1974;5(3-4):497505.

35. Sagan C, Thompson WR, Khare BN. Titan: a laboratory for prebiological organic chemistry. Accounts of Chemical Research. 2002;25(7):286-292. 


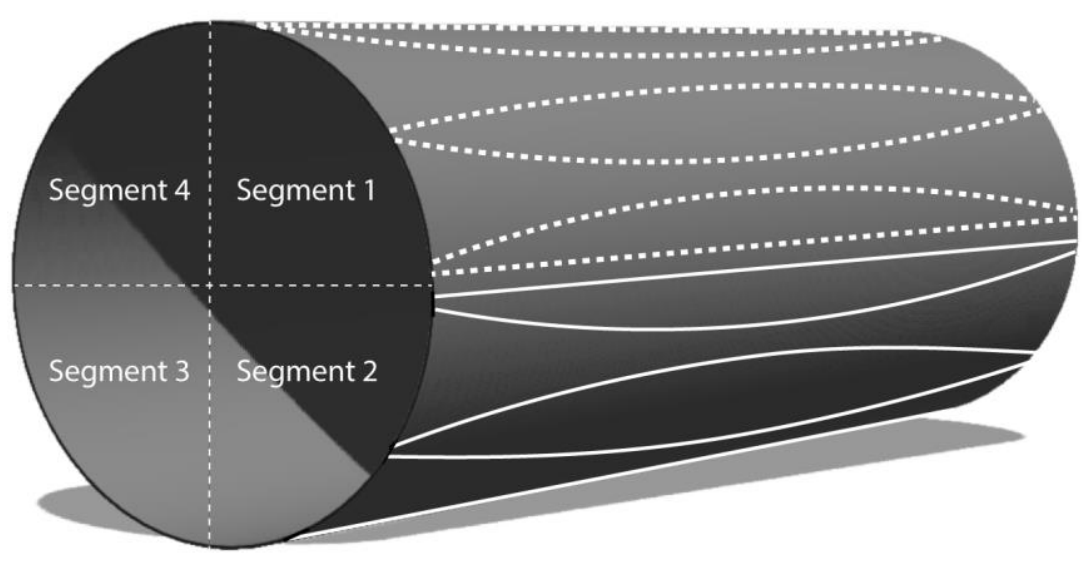

447 Figure 1: Schematic representation of a dynamically harmonized ICR cell. The cell is 448 divided into four segments representing each adjustable parameter: Segment 1 (in dashed 449 lines) is for shimming and gating $0^{\circ}$, segment 2 (in solid lines) for shimming and gating $45090^{\circ}$, segment 3 for shimming and gating $180^{\circ}$ and segment 4 for shimming and gating $270^{\circ}$. 

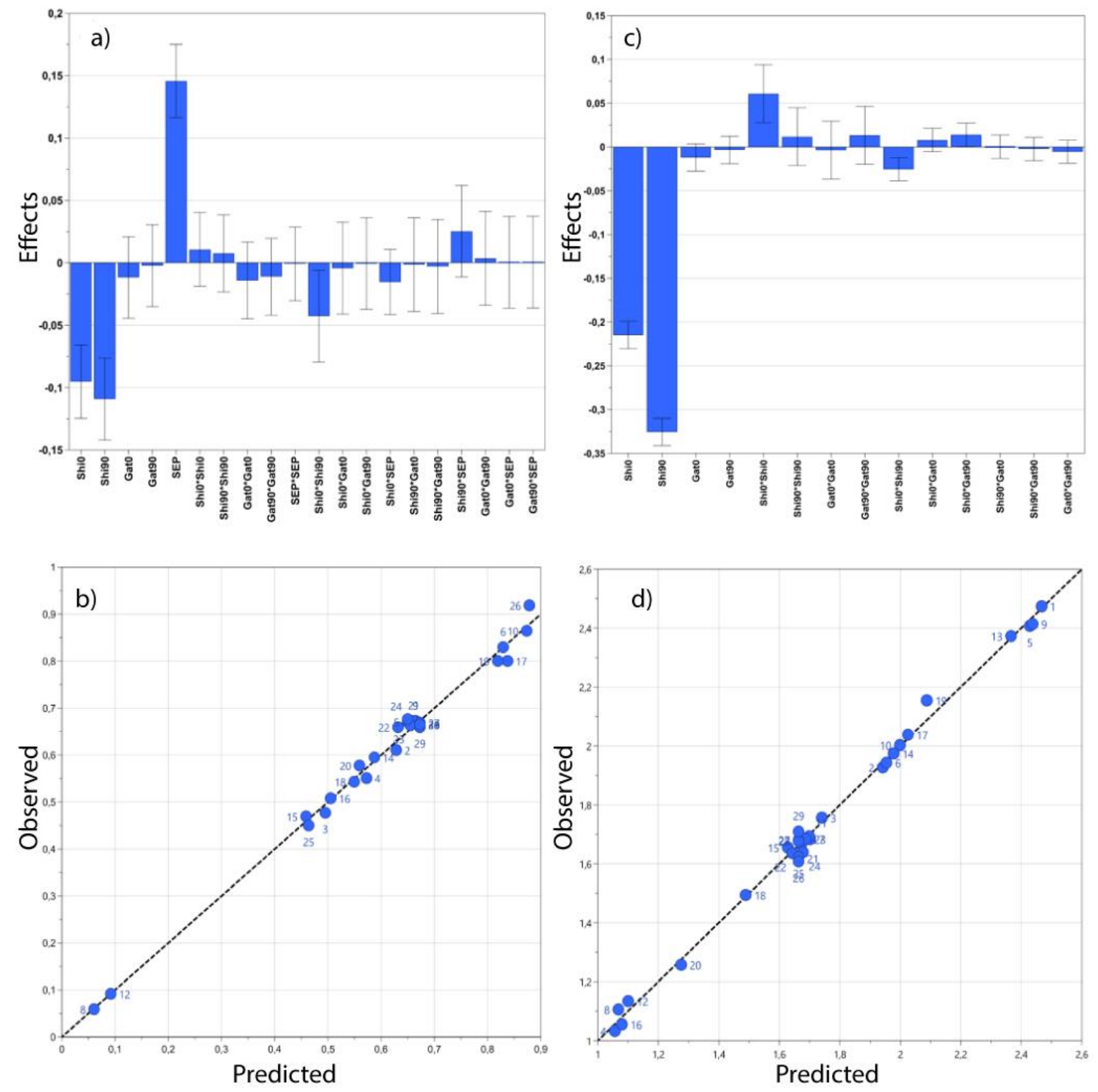

454 Figure 2: a) Observation of the effects of the different factors on the even harmonic 455 magnitude for the screening step, b) Prediction plot between the predicted and observed 456 values of the second harmonic magnitude for the screening step, c) Observation of the 457 effects of the different factors on the second harmonic magnitude for the optimization step, 458 d) Prediction plot between the predicted and observed values of the second harmonic 459 magnitude for the optimization step. 


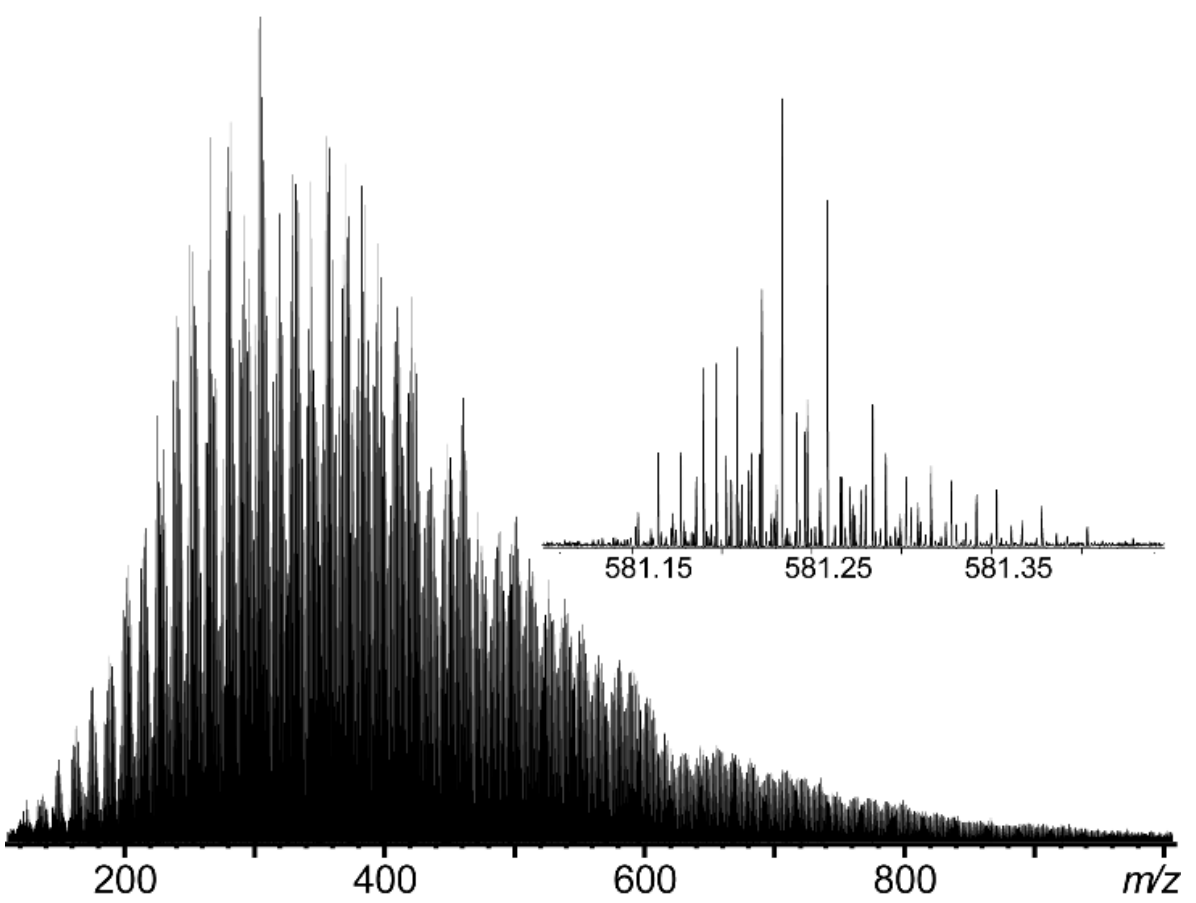

463 Figure 3: LDI spectrum in positive mode of a tholins sample obtained after deploying the 464 cell parameter optimized with the DoE approach, revealing more than 50,000 peaks. The 465 inset visualized a close zoom between $\mathrm{m} / \mathrm{z} .581 .15$ and $\mathrm{m} / \mathrm{z} .581 .40$ illustrating the high 466 isobaric complexity.

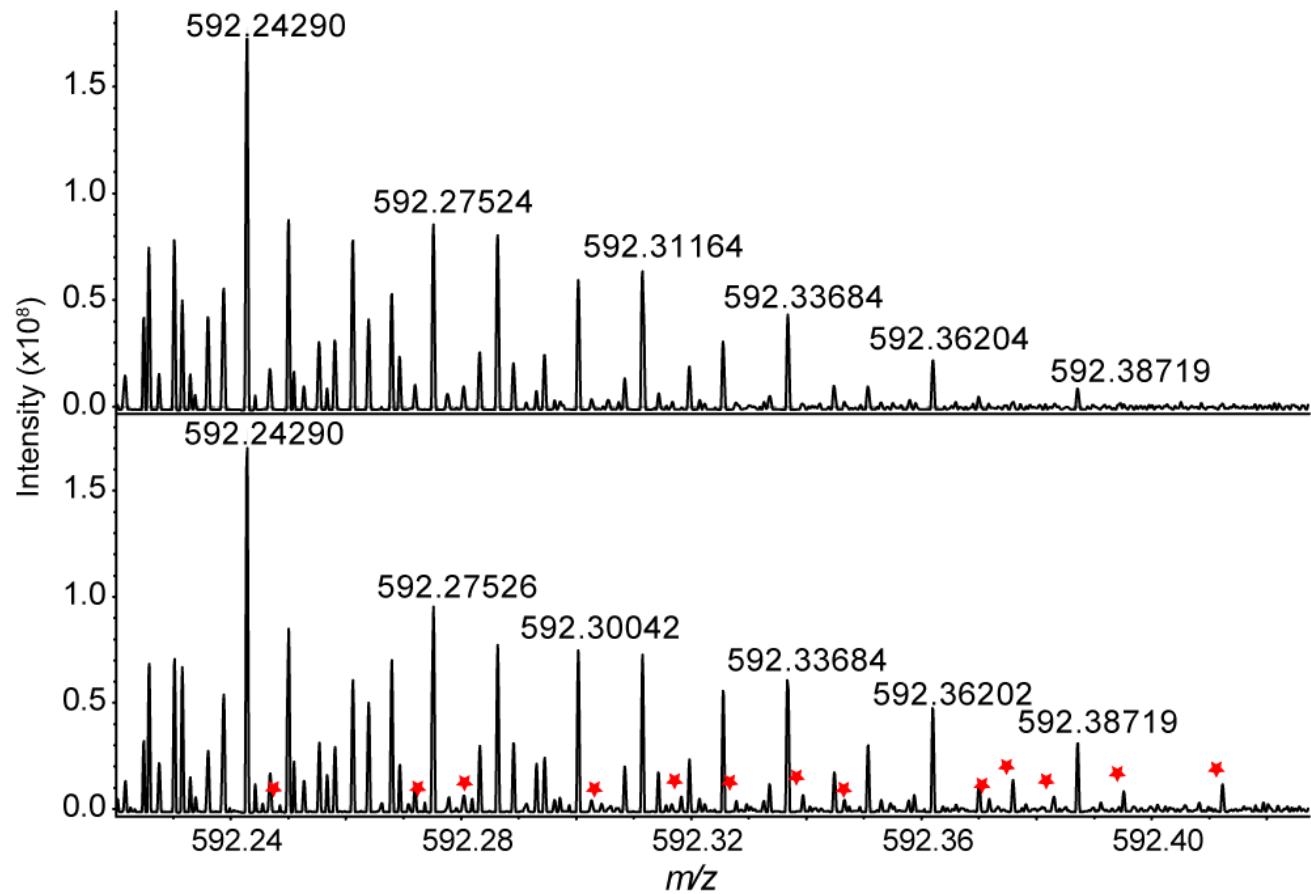

468 Figure 4: (Top) Zoom on the tholins spectrum obtained after a manual optimization 469 (shimming) of the ICR cell parameters. (Bottom) Tholins spectrum obtained after applying 470 the parameters given by the DoE optimization. Red dots indicate species found after DoE 471 optimization and not detected after manual optimization. 


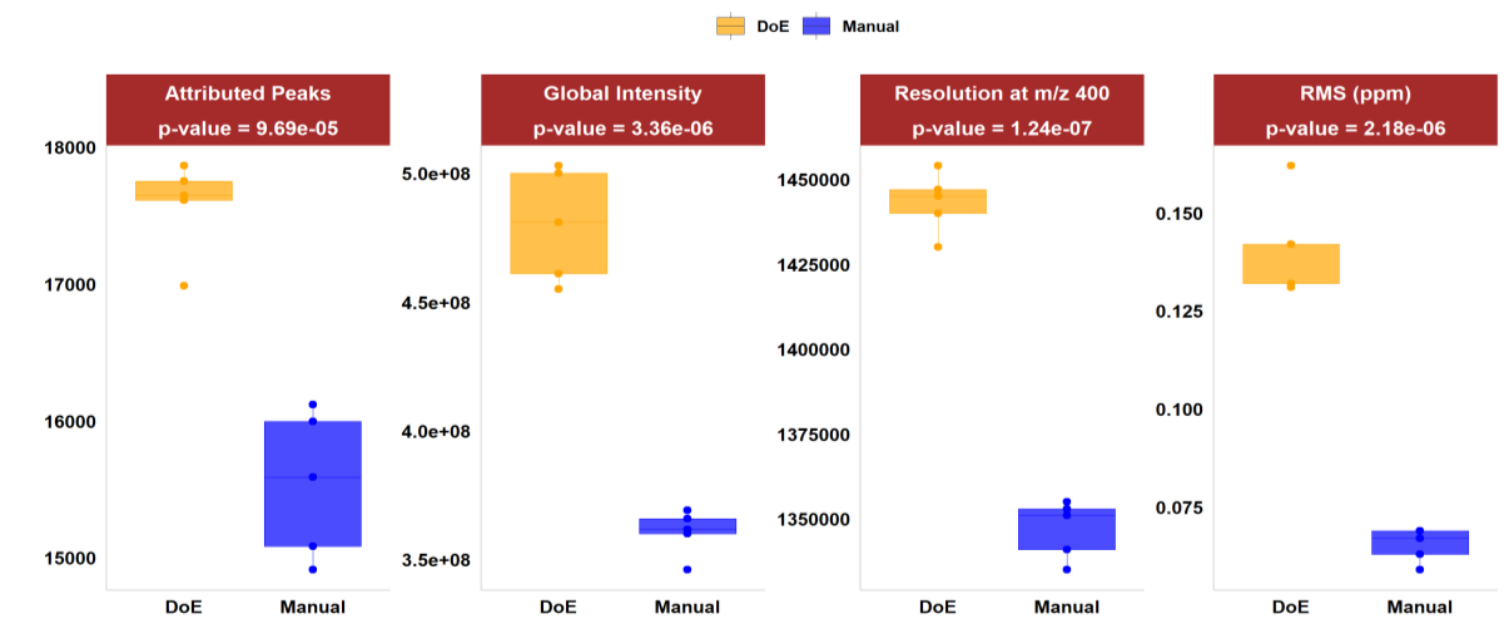

473

474

475

476

477

Table 1: Coded composite face design (CCF) design matrix for experimental

478 screening on NaTFA
Figure 5: Comparison of attributed peaks, global intensity, resolution and RMS between the manually optimized spectrum and the DoE spectrum of a tholins sample.

\begin{tabular}{|c|c|c|c|c|c|c|}
\hline Run $\mathbf{N}^{\circ}$ & Run Order & Shio & Shi90 & Gat0 & Gat90 & SEP \\
\hline 1 & 10 & -1 & -1 & -1 & -1 & 1 \\
\hline 2 & 28 & 1 & -1 & -1 & -1 & -1 \\
\hline 3 & 23 & -1 & 1 & -1 & -1 & -1 \\
\hline 4 & 9 & 1 & 1 & -1 & -1 & 1 \\
\hline 5 & 1 & -1 & -1 & 1 & -1 & -1 \\
\hline 6 & 24 & 1 & -1 & 1 & -1 & 1 \\
\hline 7 & 20 & -1 & 1 & 1 & -1 & 1 \\
\hline 8 & 12 & 1 & 1 & 1 & -1 & -1 \\
\hline 9 & 29 & -1 & -1 & -1 & 1 & -1 \\
\hline 10 & 8 & 1 & -1 & -1 & 1 & 1 \\
\hline 11 & 6 & -1 & 1 & -1 & 1 & 1 \\
\hline 12 & 5 & 1 & 1 & -1 & 1 & -1 \\
\hline 13 & 25 & -1 & -1 & 1 & 1 & 1 \\
\hline 14 & 16 & 1 & -1 & 1 & 1 & -1 \\
\hline 15 & 26 & -1 & 1 & 1 & 1 & -1 \\
\hline 16 & 17 & 1 & 1 & 1 & 1 & 1 \\
\hline 17 & 2 & -1 & 0 & 0 & 0 & 0 \\
\hline 18 & 13 & 1 & 0 & 0 & 0 & 0 \\
\hline 19 & 15 & 0 & -1 & 0 & 0 & 0 \\
\hline 20 & 19 & 0 & 1 & 0 & 0 & 0 \\
\hline 21 & 4 & 0 & 0 & -1 & 0 & 0 \\
\hline 22 & 14 & 0 & 0 & 1 & 0 & 0 \\
\hline 23 & 18 & 0 & 0 & 0 & -1 & 0 \\
\hline 24 & 22 & 0 & 0 & 0 & 1 & 0 \\
\hline 25 & 3 & 0 & 0 & 0 & 0 & -1 \\
\hline 26 & 7 & 0 & 0 & 0 & 0 & 1 \\
\hline 27 & 27 & 0 & 0 & 0 & 0 & 0 \\
\hline 28 & 31 & 0 & 0 & 0 & 0 & 0 \\
\hline 29 & 30 & 0 & 0 & 0 & 0 & 0 \\
\hline 30 & 11 & 0 & 0 & 0 & 0 & 0 \\
\hline 31 & 21 & 0 & 0 & 0 & 0 & 0 \\
\hline
\end{tabular}


479

480 
DoE strategy. ( $p$-value $>0.05$ is considered as significant)

\begin{tabular}{lllll}
\hline & DoE (Mean) & Manual (Mean) & p-value & Fold Change \\
\hline Attributed Peaks & $1.7610^{+04}$ & $1.5510^{+04}$ & $9.6910^{-05}$ & 1.13 \\
Global Intensity & $4.8010^{+08}$ & $3.6110^{+08}$ & $3.3610^{-06}$ & 1.33 \\
Resolution at $\mathrm{m} / \mathbf{z} 400$ & $1.4410^{+06}$ & $1.3510^{+06}$ & $1.2410^{-07}$ & 1.07 \\
RMS (ppm) & 0.140 & 0.0654 & $2.1810^{-06}$ & 2.14
\end{tabular}

Table 2: Predicted values for each factor for the screening and optimization step

\begin{tabular}{|c|c|c|c|}
\hline \multicolumn{4}{|c|}{ Screening step } \\
\hline Factor & Intervals & Predicted Value & Factor contribution (\%) \\
\hline Shim0 & 1.465 to $1.535(\mathrm{~V})$ & $1.535 \mathrm{~V}$ & 15.4 \\
\hline Shim90 & 1.465 to $1.540(\mathrm{~V})$ & $1.540 \mathrm{~V}$ & 33.3 \\
\hline Gat0 & 1.4 to $1.620(\mathrm{~V})$ & $1.620 \mathrm{~V}$ & 12.6 \\
\hline Gat90 & 1.450 to $1.550(\mathrm{~V})$ & $1.550 \mathrm{~V}$ & 10.7 \\
\hline Sweep & 20 to $40(\%)$ & $20 \%$ & 28.0 \\
\hline \multicolumn{4}{|c|}{ Optimization step } \\
\hline Factor & Intervals & Predicted Value & Factor contribution (\%) \\
\hline Shim0 & 1.530 to $1.550(\mathrm{~V})$ & $1.542 \mathrm{~V}$ & 53.1 \\
\hline Shim90 & 1.530 to $1.550(\mathrm{~V})$ & $1.550 \mathrm{~V}$ & 43.4 \\
\hline Gat0 & 1.600 to $1.640(\mathrm{~V})$ & $1.600 \mathrm{~V}$ & 1.9 \\
\hline Gat90 & 1.540 to $1.570(\mathrm{~V})$ & $1.551 \mathrm{~V}$ & 1.7 \\
\hline
\end{tabular}

Table 3. Comparison of the mass spectrometric response of the LDI tholins spectra deploying manufacturer default values (before tuning), conventional manual tuning (manual), and parameters given by the DoE optimization method. p-values refers to the comparison between manual Tuning and the

498 


\section{Supplementary Information for}

501 Optimization of ion trajectories in a dynamically harmonized

502 Fourier-Transform Ion Cyclotron Resonance cell using a Design of

503 Experiments strategy

504

505

506

507

508

509

510

511

512

513

Julien MAILLARD ${ }^{1,2}$, Justine FEREY ${ }^{2}$, Christopher P. Rüger ${ }^{2}$, Isabelle SCHMITZAFONSO $^{2}$, Soumeya BEKRI ${ }^{3}$, Thomas GAUTIER ${ }^{1}$, Nathalie CARRASCO ${ }^{1}$, Carlos $\mathrm{AFONSO}^{2}$ and Abdellah TEBANI ${ }^{2.3^{*}}$

${ }^{1}$ LATMOS/IPSL, Université Versailles St Quentin, UPMC Université Paris 06, CNRS, 11 blvd d'Alembert, F-78280 Guyancourt, France

${ }^{2}$ Université de Rouen, Laboratoire COBRA UMR 6014 \& FR 3038, IRCOF, 1 Rue Tesnière, 76821 Mont St Aignan Cedex, France

${ }^{3}$ Department of Metabolic Biochemistry, Rouen University Hospital, Rouen, 76000, France

\section{This PDF file includes:}

Tables S-1 to S-6

\section{Captions:}

- Table S-1: Fractional Factorial design matrix with response values for the screening step.

- Table S-2: Fractional Factorial design matrix with response values for the optimization step.

- Table S-3: PLS model regression coefficients generated by the screening step.

- Table S-4: ANOVA test results of the PLS model generated by the screening step.

- Table S-5: PLS model regression coefficients generated by the optimization step.

- Table S-6: ANOVA test results of the PLS model generated by the optimization step. 
Table S-1: Fractional Factorial design matrix with response values the screening step.

\begin{tabular}{|c|c|c|c|c|c|c|c|}
\hline $\begin{array}{l}\text { Run } \\
\mathbf{N}^{\circ}\end{array}$ & $\begin{array}{l}\text { Run } \\
\text { Order }\end{array}$ & $\begin{array}{c}\text { Shimo } \\
\text { (V) }\end{array}$ & $\begin{array}{c}\text { Shim90 } \\
\text { (V) }\end{array}$ & $\begin{array}{c}\text { Gat0 } \\
\text { (V) }\end{array}$ & $\begin{array}{c}\text { Gat90 } \\
\text { (V) }\end{array}$ & $\begin{array}{l}\text { SEP } \\
(\%)\end{array}$ & $\begin{array}{c}\text { Second } \\
\text { harmonic } \\
(\%)\end{array}$ \\
\hline 1 & 10 & 1.465 & 1.465 & 1.400 & 1.450 & 40 & \\
\hline 2 & 28 & 1.535 & 1.465 & 1.400 & 1.450 & 20 & 4.083 \\
\hline 3 & 23 & 1.465 & 1.540 & 1.400 & 1.450 & 20 & 2.996 \\
\hline 4 & 9 & 1.535 & 1.540 & 1.400 & 1.450 & 40 & 3.558 \\
\hline 5 & 1 & 1.465 & 1.465 & 1.620 & 1.450 & 20 & 4.694 \\
\hline 6 & 24 & 1.535 & 1.465 & 1.620 & 1.450 & 40 & 6.77 \\
\hline 7 & 20 & 1.465 & 1.540 & 1.620 & 1.450 & 40 & \\
\hline 8 & 12 & 1.535 & 1.540 & 1.620 & 1.450 & 20 & 1.143 \\
\hline 9 & 29 & 1.465 & 1.465 & 1.400 & 1.550 & 20 & 4.714 \\
\hline 10 & 8 & 1.535 & 1.465 & 1.400 & 1.550 & 40 & 7.312 \\
\hline 11 & 6 & 1.465 & 1.540 & 1.400 & 1.550 & 40 & \\
\hline 12 & 5 & 1.535 & 1.540 & 1.400 & 1.550 & 20 & 1.237 \\
\hline 13 & 25 & 1.465 & 1.465 & 1.620 & 1.550 & 40 & \\
\hline 14 & 16 & 1.535 & 1.465 & 1.620 & 1.550 & 20 & 3.937 \\
\hline 15 & 26 & 1.465 & 1.540 & 1.620 & 1.550 & 20 & 2.946 \\
\hline 16 & 17 & 1.535 & 1.540 & 1.620 & 1.550 & 40 & 3.228 \\
\hline 17 & 2 & 1.465 & 1.503 & 1.510 & 1.500 & 30 & 6.332 \\
\hline 18 & 13 & 1.535 & 1.503 & 1.510 & 1.500 & 30 & 3.494 \\
\hline 19 & 15 & 1.500 & 1.465 & 1.510 & 1.500 & 30 & 6.305 \\
\hline 20 & 19 & 1.500 & 1.540 & 1.510 & 1.500 & 30 & 3.788 \\
\hline 21 & 4 & 1.500 & 1.503 & 1.400 & 1.500 & 30 & 4.716 \\
\hline 22 & 14 & 1.500 & 1.503 & 1.620 & 1.500 & 30 & 4.56 \\
\hline 23 & 18 & 1.500 & 1.503 & 1.510 & 1.450 & 30 & 4.599 \\
\hline 24 & 22 & 1.500 & 1.503 & 1.510 & 1.550 & 30 & 4.747 \\
\hline 25 & 3 & 1.500 & 1.503 & 1.510 & 1.500 & 20 & 2.819 \\
\hline 26 & 7 & 1.500 & 1.503 & 1.510 & 1.500 & 40 & 8.281 \\
\hline 27 & 27 & 1.500 & 1.503 & 1.510 & 1.500 & 30 & 4.659 \\
\hline 28 & 31 & 1.500 & 1.503 & 1.510 & 1.500 & 30 & 4.627 \\
\hline 29 & 30 & 1.500 & 1.503 & 1.510 & 1.500 & 30 & 4.567 \\
\hline 30 & 11 & 1.500 & 1.503 & 1.510 & 1.500 & 30 & 4.629 \\
\hline 31 & 21 & 1.500 & 1.503 & 1.510 & 1.500 & 30 & 4.624 \\
\hline
\end{tabular}

527 
Table S-2: Fractional Factorial design matrix with response values for the optimization step.

\begin{tabular}{ccccccc}
\hline $\begin{array}{c}\text { Run } \\
\mathbf{N}^{\circ}\end{array}$ & $\begin{array}{c}\text { Run } \\
\text { Order }\end{array}$ & $\begin{array}{c}\text { Shim0 } \\
(\mathbf{V})\end{array}$ & $\begin{array}{c}\text { Shim90 }(\mathbf{V}) \\
1\end{array}$ & $\begin{array}{c}\text { Gat0 } \\
(\mathbf{V})\end{array}$ & $\begin{array}{c}\text { Gat90 } \\
(\mathbf{V})\end{array}$ & $\begin{array}{c}\text { Second harmonic } \\
(\%)\end{array}$ \\
2 & 27 & 1.530 & 1.530 & 1.600 & 1.540 & 2.475 \\
3 & 2 & 1.550 & 1.530 & 1.600 & 1.540 & 1.928 \\
4 & 20 & 1.530 & 1.550 & 1.600 & 1.540 & 1.757 \\
5 & 4 & 1.550 & 1.550 & 1.600 & 1.540 & 1.033 \\
6 & 5 & 1.530 & 1.530 & 1.640 & 1.540 & 2.407 \\
7 & 23 & 1.550 & 1.530 & 1.640 & 1.540 & 1.942 \\
8 & 12 & 1.530 & 1.550 & 1.640 & 1.540 & 1.684 \\
9 & 24 & 1.550 & 1.550 & 1.640 & 1.540 & 1.107 \\
10 & 19 & 1.530 & 1.530 & 1.600 & 1.570 & 2.414 \\
11 & 6 & 1.550 & 1.530 & 1.600 & 1.570 & 2.003 \\
12 & 26 & 1.530 & 1.550 & 1.600 & 1.570 & 1.693 \\
13 & 3 & 1.550 & 1.550 & 1.600 & 1.570 & 1.135 \\
14 & 1 & 1.530 & 1.530 & 1.640 & 1.570 & 2.373 \\
15 & 28 & 1.550 & 1.530 & 1.640 & 1.570 & 1.975 \\
16 & 29 & 1.550 & 1.550 & 1.640 & 1.570 & 1.654 \\
17 & 17 & 1.530 & 1.540 & 1.640 & 1.570 & 1.054 \\
18 & 18 & 1.550 & 1.540 & 1.620 & 1.555 & 2.039 \\
19 & 8 & 1.540 & 1.530 & 1.620 & 1.555 & 1.493 \\
20 & 10 & 1.540 & 1.550 & 1.620 & 1.555 & 2.156 \\
21 & 21 & 1.540 & 1.540 & 1.600 & 1.555 & 1.256 \\
22 & 22 & 1.540 & 1.540 & 1.640 & 1.555 & 1.665 \\
23 & 16 & 1.540 & 1.540 & 1.620 & 1.540 & 1.637 \\
24 & 15 & 1.540 & 1.540 & 1.620 & 1.570 & 1.684 \\
25 & 25 & 1.540 & 1.540 & 1.620 & 1.555 & 1.639 \\
26 & 14 & 1.540 & 1.540 & 1.620 & 1.555 & 1.623 \\
27 & 13 & 1.540 & 1.540 & 1.620 & 1.555 & 1.608 \\
28 & 7 & 1.540 & 1.540 & 1.620 & 1.555 & 1.682 \\
29 & 9 & 1.540 & 1.540 & 1.620 & 1.555 & 1.676 \\
\hline & & & & & & 1.708 \\
\hline
\end{tabular}




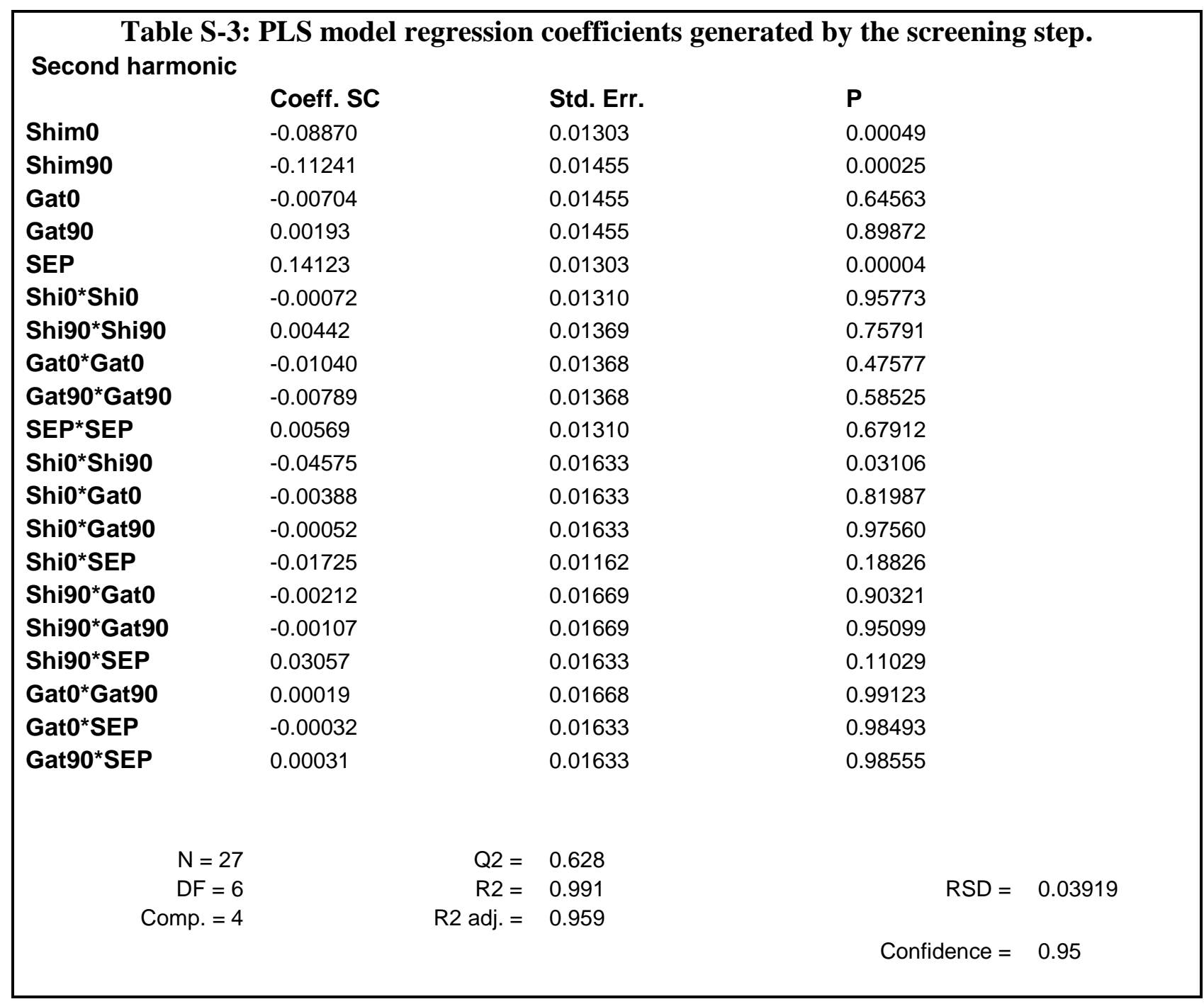

\begin{tabular}{|c|c|c|c|c|c|}
\hline \multicolumn{6}{|c|}{$\begin{array}{l}\text { Table S-4: ANOVA test results of the PLS model generated by the screening step. } \\
\text { Second } \\
\text { harmonic }\end{array}$} \\
\hline & DF & SS & MS (variance) & $\mathbf{F}$ & $p$ \\
\hline Total & 27 & 11.0417 & 0.40895 & & \\
\hline Total corrected & 26 & 0.972344 & 0.0373979 & & \\
\hline Regression & 20 & 0.96313 & 0.0481565 & 31.3577 & 0 \\
\hline \multirow[t]{4}{*}{ Residual } & 6 & 0.00921428 & 0.00153571 & & \\
\hline & $N=27$ & Q2 = & 0.628 & Cond. no. = & 12.05 \\
\hline & $\mathrm{DF}=6$ & $\mathrm{R} 2=$ & 0.991 & $\mathrm{RSD}=$ & $\begin{array}{l}0.039 \\
19\end{array}$ \\
\hline & Comp. $=4$ & $\mathrm{R} 2 \mathrm{adj} .=$ & 0.959 & & \\
\hline
\end{tabular}




\begin{tabular}{|c|c|c|c|c|}
\hline \multicolumn{5}{|c|}{$\begin{array}{l}\text { Table S-5: PLS model regression coefficients generated by the optimization step. } \\
\text { Second } \\
\text { harmonic }\end{array}$} \\
\hline & Coeff. SC & Std. Err. & $\mathbf{P}$ & \\
\hline Constant & 1,66208 & 0,0126069 & $4,58 \mathrm{E}-23$ & \\
\hline Shimo & $-0,214759$ & 0,0072904 & $5,37 E-14$ & \\
\hline Shim90 & $-0,325479$ & 0,00729039 & $1,69 \mathrm{E}-16$ & \\
\hline Gat0 & $-0,0120755$ & 0,0072904 & 0,119882 & \\
\hline Gat90 & $-0,00339587$ & 0,0072904 & 0,648523 & \\
\hline Shi0*ShiO & 0,0608795 & 0,0154223 & 0,00145892 & \\
\hline Shi90*Shi90 & 0,0117455 & 0,0154223 & 0,458943 & \\
\hline Gat0*Gat0 & $-0,00371134$ & 0,0154223 & 0,813317 & \\
\hline Gat90*Gat90 & 0,0132228 & 0,0154223 & 0,405672 & \\
\hline Shi0*Shi90 & $-0,0255319$ & 0,00619991 & 0,00104456 & \\
\hline Shio*Gat0 & 0,00798444 & 0,0061999 & 0,218685 & \\
\hline Shio*Gat9o & 0,0138399 & 0,0061999 & 0,0424476 & \\
\hline Shi90*Gat0 & 0,000118387 & 0,0061999 & 0,985034 & \\
\hline Shi90*Gat90 & $-0,0022854$ & 0,0061999 & 0,717926 & \\
\hline Gat0*Gat90 & $-0,00542158$ & 0,0061999 & 0,39662 & \\
\hline$N=29$ & $\mathrm{Q} 2=$ & 0,889 & & \\
\hline $\mathrm{DF}=14$ & $\mathrm{R} 2=$ & 0,995 & $\mathrm{RSD}=$ & 0,03858 \\
\hline Comp. $=5$ & $\mathrm{R} 2 \mathrm{adj} .=$ & 0,991 & & \\
\hline & & & Confidence $=$ & 0,95 \\
\hline
\end{tabular}

Table S-6: ANOVA test results of the PLS model generated by the optimization step.

\begin{tabular}{|c|c|c|c|c|c|}
\hline \multicolumn{6}{|l|}{$\begin{array}{l}\text { Second } \\
\text { harmonic }\end{array}$} \\
\hline Total & 29 & 92,3573 & 3,18473 & & \\
\hline Total corrected & 28 & 4,41765 & 0,157773 & & \\
\hline Regression & 14 & 4,39681 & 0,314058 & 211,033 & 0 \\
\hline Residual & 14 & 0,0208347 & 0,00148819 & & \\
\hline & $N=29$ & Q2 = & 0,889 & Cond. no. = & 6,106 \\
\hline & $\mathrm{DF}=14$ & $\mathrm{R} 2=$ & 0,995 & $\mathrm{RSD}=$ & 0,03858 \\
\hline & Comp. $=5$ & $\mathrm{R} 2 \mathrm{adj} .=$ & 0,991 & & \\
\hline
\end{tabular}

536 
538 Table S-7: Attribution of new peaks detected after the DoE optimization

539

\begin{tabular}{c|c|c}
\hline Measured $\boldsymbol{m} / \boldsymbol{z}$ & Molecular formula & Err. (ppm) \\
\hline 592.24866 & $\mathrm{C} 25 \mathrm{H} 30 \mathrm{~N} 1305$ & 0.13 \\
\hline 592.27374 & $\mathrm{C} 26 \mathrm{H} 32 \mathrm{~N} 1303^{\wedge} 18 \mathrm{O}$ & -0.04 \\
\hline 592.28203 & $\mathrm{C} 27 \mathrm{H} 35 \mathrm{~N} 10 \mathrm{O} 5^{\wedge} 13 \mathrm{C}$ & -0.05 \\
\hline 592.31836 & $\mathrm{C} 38 \mathrm{H} 38 \mathrm{~N} 7$ & -0.06 \\
\hline 592.32791 & $\mathrm{C} 19 \mathrm{H} 41 \mathrm{~N} 1407^{\wedge} 15 \mathrm{~N}$ & -0.14 \\
\hline 592.33949 & $\mathrm{C} 25 \mathrm{H} 43 \mathrm{~N} 1006^{\wedge} 13 \mathrm{C}$ & 0.03 \\
\hline 592.34673 & $\mathrm{C} 19 \mathrm{H} 43 \mathrm{~N} 1407^{\wedge} 13 \mathrm{C}$ & -0.01 \\
\hline 592.37013 & $\mathrm{C} 33 \mathrm{H} 43 \mathrm{~N} 10^{\wedge} 13 \mathrm{C}$ & -0.11 \\
\hline 592.37597 & $\mathrm{C} 26 \mathrm{H} 47 \mathrm{~N} 1005^{\wedge} 13 \mathrm{C}$ & -0.12 \\
\hline 592.38314 & $\mathrm{C} 30 \mathrm{H} 46 \mathrm{~N} 1102$ & -0.17 \\
\hline 592.39528 & $\mathrm{C} 35 \mathrm{H} 47 \mathrm{~N} 8^{\wedge} 13 \mathrm{C}$ & -0.17 \\
\hline 592.41238 & $\mathrm{C} 37 \mathrm{H} 50 \mathrm{~N} 7$ & -0.11 \\
\hline
\end{tabular}

